\title{
Pharmacokinetics of ropivacaine in elderly patients receiving fascia iliaca compartment block
}

\author{
FANG-FANG ZHANG, CHEN LV, LIU-YING YANG, SHI-PING WANG, \\ MEI ZHANG and XIAO-WEN GUO \\ Department of Anesthesiology, The First Affiliated Hospital of Zhejiang Chinese Medical University, \\ Hangzhou, Zhejiang 310006, P.R. China
}

Received July 3, 2018; Accepted July 23, 2019

DOI: $10.3892 /$ etm.2019.7838

\begin{abstract}
Local anesthetic of ropivacaine was demonstrated to reduce the postoperative pain in elderly patients. This study investigated the pharmacokinetics of ropivacaine at different concentrations in elderly patients subjected to fascia iliaca compartment block. Forty patients with femoral neck fracture at American Society of Anesthesiologists (ASA) I-II status, undergoing fascia iliaca compartment block (FICB) were randomized to two groups receiving $0.7 \mathrm{ml} / \mathrm{kg}$ of solution containing $0.375 \%$ ropivacaine (group L) or $0.5 \%$ ropivacaine (group $\mathrm{H}$ ). Samples of venous blood were obtained immediately at different time points after FICB, and the total and free plasma concentrations of ropivacaine were measured by liquid chromatography-electrospray ionization-tandem mass spectrometry (LC-ESI-MS/MS). Statistical analysis was carried out using a pharmacokinetic calculation program (DAS 3.0). Visual Analogue Scale (VAS) scores were significantly decreased after FICB in both groups, and VAS score in group $\mathrm{H}$ was lower compared with group $\mathrm{L}$. The total maximum plasma concentration $\left(\mathrm{C}_{\max }\right)$ and the free $\mathrm{C}_{\max }$ of ropivacaine in group $\mathrm{H}$ was higher than that in group $\mathrm{L}(\mathrm{P}<0.05)$. The decrease of the total and free plasma concentration was operation time-dependent. Neither group showed signs of central nervous system and circulatory system toxicity. On the basis of these results, the concentrations of 0.375 and $0.5 \%$ ropivacaine held an efficiently analgesic effect for FICB, suggesting that ropivacaine can be employed in analgesic therapy. However, both concentrations have a potentially theoretical risk of local anesthetics poisoning, suggesting that a lower concentration may be a safer option for a single large volume of FICB.
\end{abstract}

Correspondence to: Dr Xiao-Wen Guo, Department of Anesthesiology, The First Affiliated Hospital of Zhejiang Chinese Medical University, 54 Youdian Road, Shangcheng, Hangzhou, Zhejiang 310006, P.R. China

E-mail: guoxiaowen13152257@163.com

Key words: fascia iliaca compartment bloc, ropivacaine, pharmacokinetics, local anesthetic, toxicity, femoral neck fracture

\section{Introduction}

In emergency medical treatment, patients with femoral neck fracture often suffer from severe pain, resulting in some complications, especially in the elderly. In response to this, several non-steroidal anti-inflammatory drugs (NSAIDs), including acetaminophen, have been applied to control the pain (1), although the actual effect is not satisfying. NSAIDs can cause cardio-renal toxicity (2), gastrointestinal bleeding (3) and platelet inhibition $(4,5)$. In addition, it has been demonstrated that the application of opioids, such as fentanyl and morphine, often cause nausea and vomiting, as well as other common side-effects, including excessive sedation, respiratory depression and delirious symptoms (6-9). Up to now, for most of the patients in the emergency room effective and secure analgesia were unavailable, which was one of the factors of acute deliria (10).

Fascia iliaca compartment block (FICB) has been reported to provide a more extensive blocking range and better analgesic effect compared with the three-in-one femoral nerve block $(11,12)$. FICB has been widely used in perioperative analgesia by anesthesiologists with a good effect $(13,14)$. Randomized controlled trials (RCTs) and low quality results have suggested that FICB could be well used in the management of acute pain in patients with femoral neck fracture (15-17). However, in a previous clinical study it was reported that the analgesic effect of FICB mainly depends on the capacity of the local anesthetic solution. Occasionally, in order to obtain a longer duration of analgesia, a higher concentration of local anesthetics has been considered to apply clinically. However, the incidence of local anesthetic systemic toxicity (LAST) may increase with the dose increase (18).

Ropivacaine, a stereo-specific levorotary local anesthetic, has been widely utilized in peripheral nerve block analgesia (19). Compared with bupivacaine, ropivacaine exerts vasoconstrictor effects and has a lower cardiac toxicity risk, and therefore has been widely used in surgical analgesia $(20,21)$. A previous report by Paut et al (22) showed that a maximum plasma concentration $\left(\mathrm{C}_{\max }\right.$ of $>50 \%$ in children with FICB is more than twice the alert concentration of ropivacaine $(2.2 \mu \mathrm{g} / \mathrm{ml})$, without the occurrence of serious LAST, indicating that FICB may have a higher risk of LAST in specific populations. Also, a case report revealed that a severe 
LAST occurred after the interscalene brachial plexus block with $75 \mathrm{mg}$ ropivacaine in a 76-year old female patient (23). In addition to the local anesthetic dose, the occurrence of LAST is closely related to the blood flow of the injection region. However, it has been reported that compared with young adults, elderly patients have poor blood vessels in tissue surrounding the nerves, thus, a slower absorption of local anesthetic in the elderly causes a low $\mathrm{C}_{\max }$ and prolonged time to reach the maximum plasma concentration $\left(\mathrm{T}_{\max }\right)(24,25)$. Free plasma concentration of local anesthetic is known to be the key factor of LAST. Torup et al (26) have reported that although the total plasma concentration of ropivacaine of $\sim 30 \%$ in patients exceeded the alert of neurotoxicity after bilateral transverse fascia block, no toxicity reaction occurred clinically, as the free plasma concentration was below the warning level. Therefore, the effective and safe dose of ropivacaine in patients, and especially the elderly, is important to be investigated comprehensively.

\section{Patients and methods}

Case selection and grouping. After obtaining the approval from the Ethics Committee of the First Affiliated Hospital of Zhejiang Chinese Medicine University (Hangzhou, China) (no. 2013-k-058), 40 patients at American Society of Anesthesiologists (ASA) I-II stage with femoral neck fracture, 60-85 years of age, were enrolled in the study. Patients with the following conditions were excluded from the study: patients under treatment for chronic pain; with local anesthetic allergy; with injection site infection; body mass index $>30 \mathrm{~kg} / \mathrm{m}^{2}$; lower extremity nerve disease; comprehension barriers on the Visual Analogue Scale (VAS). All the patients were informed of the study and signed a consent form. According to the random Digital Envelope method, patients were randomly assigned to receive FICB with a $0.7 \mathrm{ml} / \mathrm{kg}$ of solution containing $0.375 \%$ ropivacaine (group L) or $0.5 \%$ ropivacaine (group $\mathrm{H}$ ), as previously described (27-29).

FICB operation. In the emergency room, patients were given oxygen saturation $\left(\mathrm{SPO}_{2}\right)$, non-invasive arterial blood pressure and electrocardiograph (ECG) monitoring and recording were carried out, and vein channels were established. A needle was placed $1 \mathrm{~cm}$ below one-third the distance from the pubic tubercle toward the anterior superior iliac spine (ASIS). The skin was pierced with a large diameter needle and then inserted into a blunt needle (16G Tuohy) at 45 degrees until iliac fascia. Prior to the infusion of ropivacaine, the position of the needle was confirmed by ultrasound. Subsequently, group L received $0.7 \mathrm{ml} / \mathrm{kg}$ of $0.375 \%$ ropivacaine (Naropine ${ }^{\circledR}$; AstraZeneca AB), and group $\mathrm{H}$ received $0.7 \mathrm{ml} / \mathrm{kg}$ of $0.5 \%$ ropivacaine.

VAS scoring. Pain was evaluated based on VAS, with 0 characterizing no pain and 10 used for the most intense pain. VAS scores were recorded before and at 20 min after the block. To guarantee the objectivity of the data, VAS and block were assessed in a blinded manner to patient treatment, and statistical analysis was carried out after the separate assessments.

Block scope assessment. The block range of lateral, anterior and medial thigh, corresponding to the lateral femoral cutaneous
Table I. General characteristics of patients in both groups.

\begin{tabular}{lccc}
\hline Variables & Group L $(\mathrm{n}=20)$ & Group H $(\mathrm{n}=20)$ & P-value \\
\hline Age (years) & $76.4 \pm 5.6$ & $73.8 \pm 6.3$ & 0.176 \\
Sex (M/F) & $8 / 12$ & $10 / 10$ & 0.751 \\
Weight (kg) & $57.1 \pm 10.8$ & $62.3 \pm 8.6$ & 0.100 \\
Height (cm) & $162.5 \pm 8.3$ & $160.9 \pm 12.6$ & 0.636 \\
ASA physical & $7 / 13$ & $5 / 15$ & 0.730 \\
status (I/II) & & &
\end{tabular}

ASA, American Society of Anesthesiologists.

nerve, femoral nerve and obturator nerve area, was tested with the cold sensation disappearance method. The detailed data on nerve blocking at the three sites were recorded at $20 \mathrm{~min}$ after the operation, including failure, in one, two or three nerve innervation areas.

Assessment of cardiovascular and nervous system toxicity. The effect of local anesthetics on auditory and/or visual impairment, perioral numbness, tingling, paresthesia and/or paralysis, muscle twitching and/or muscle stiffness, and articulation disorders, were evaluated before, and at 1 or $2 \mathrm{~h}$ after FICB operation.

Blood sample collection and testing. Samples $(3 \mathrm{ml})$ of venous blood were obtained immediately, and at 15, 30, 45, 60, 90 and $120 \mathrm{~min}$ after FICB. Plasma was isolated within an hour and frozen immediately at $-80^{\circ} \mathrm{C}$. The liquid chromatography-electrospray ionization-tandem mass spectrometry (LC-ESI-MS/MS) was applied to determine the total and free plasma concentration of ropivacaine. The free plasma concentration was obtained by equilibrium dialysis. The concentration-vs.-time curve of plasma ropivacaine was simulated using DAS 3.0 pharmacokinetic software (Anhui Provincial Drug Clinical Evaluation Center) to calculate the pharmacokinetic parameters, namely, $\mathrm{C}_{\max }, \mathrm{T}_{\max }$, elimination half-time $\left(\mathrm{T}_{1 / 2} \mathrm{z}\right)$, area under the plasma concentration-time curve $\left(\mathrm{AUC}_{0-\mathrm{t}}\right)$, area under the plasma concentration-time curve extrapolated to infinity $\left(\mathrm{AUC}_{0-\infty}\right)$, and clearance $\left(\mathrm{CL}_{\mathrm{z}} / \mathrm{F}\right)$.

Statistical analysis. SPSS 19.0 statistical software (IBM Corp.) was used for statistical analysis. All quantitative measurement data were expressed as the mean \pm standard deviation (SD) and analyzed by Student's t-test. Dichotomous data (age, sex, thigh sensory blocks) were analyzed using Chi-square test. $\mathrm{P}<0.05$ was considered to indicate a statistically significant difference.

\section{Results}

General characteristics. There was no significant difference in sex ratio, age, ASA classification, height and weight between the groups, as shown in Table I.

Effect of analgesia, block and nervous system toxicity. FICB achieved good analgesic effect in groups L and H. The VAS scores were recorded before FICB and at $20 \mathrm{~min}$ after FICB. As 
Table II. VAS scores before and at 20 min after FICB.

\begin{tabular}{lccc}
\hline Variables & Group L $(\mathrm{n}=20)$ & Group H $(\mathrm{n}=20)$ & P-value \\
\hline Before FICB & $6.30 \pm 0.97$ & $6.46 \pm 1.02$ & 0.614 \\
At 20 min & $2.87 \pm 0.73^{\mathrm{a}}$ & $2.27 \pm 0.82^{\mathrm{a}, \mathrm{b}}$ & 0.020 \\
after FICB & & & \\
\hline
\end{tabular}

${ }^{\mathrm{a}} \mathrm{P}<0.05$, compared with before FICB; ${ }^{\mathrm{b}} \mathrm{P}<0.05$, compared with group $\mathrm{L}$. VAS, Visual Analogue Scale; FICB, fascia iliaca compartment block.

Table III. Thigh sensory blocks at $20 \mathrm{~min}$ after FICB in the two groups $[\mathrm{n}(\%)]$.

Sensory block Group L (n=20) Group H (n=20) P-value

\begin{tabular}{llll}
\hline $\mathrm{LFC}+\mathrm{F}$ & $20(100.0)$ & $20(100)$ & 1.00 \\
$\mathrm{LFC}+\mathrm{F}+\mathrm{O}$ & $15(75.0)$ & $17(85)$ & 0.695
\end{tabular}

FICB, fascia iliaca compartment block; LFC, lateral femoral cutaneous; F, femoral; O, obturator.

Table IV. Comparison of the kinetic parameters of total plasma pharmacokinetics of ropivacaine in the two groups.

\begin{tabular}{lccc}
\hline Parameters & Group L (n=20) & Group H (n=20) & P-value \\
\hline $\mathrm{AUC}_{0-\mathrm{t}}(\mathrm{mg} / \mathrm{l} \mathrm{x} \mathrm{h})$ & $2.48 \pm 0.76$ & $3.36 \pm 0.63$ & $<0.01$ \\
$\mathrm{AUC}_{0-\infty}(\mathrm{mg} / \mathrm{l} \mathrm{x} \mathrm{h})$ & $7.82 \pm 1.37$ & $8.68 \pm 1.04$ & 0.034 \\
$\mathrm{t}_{1 / 2} \mathrm{Z}(\mathrm{h})$ & $2.78 \pm 1.02$ & $2.28 \pm 0.61$ & 0.072 \\
$\mathrm{~T}_{\max }(\mathrm{h})$ & $0.53 \pm 0.12$ & $0.56 \pm 0.09$ & 0.428 \\
$\mathrm{CL}_{\mathrm{z}} / \mathrm{F}\left(\mathrm{m}^{3} / \mathrm{h} / \mathrm{kg}\right)$ & $40.8 \pm 14.7$ & $38.9 \pm 12.0$ & 0.662 \\
$\mathrm{C}_{\max }(\mathrm{mg} / \mathrm{l})$ & $1.56 \pm 0.42$ & $2.17 \pm 0.56$ & $<0.01$ \\
\hline
\end{tabular}

$\mathrm{AUC}_{0-\mathrm{t}}$, area under the plasma concentration-time curve; $\mathrm{AUC}_{0-\infty}$, area under the plasma concentration-time curve extrapolated to infinity; $\mathrm{T}_{1 / 2} \mathrm{Z}$, elimination half-time; $\mathrm{T}_{\max }$, time to reach the maximum plasma concentration; $\mathrm{CL}_{\mathrm{z}} / \mathrm{F}$, clearance; $\mathrm{C}_{\max }$, maximum plasma concentration.

Table V. Comparison of the kinetic parameters of free plasma pharmacokinetics of ropivacaine in the two groups.

\begin{tabular}{lccc}
\hline Parameters & Group L $(\mathrm{n}=20)$ & Group H $(\mathrm{n}=20)$ & P-value \\
\hline $\mathrm{AUC}_{0-\mathrm{t}}(\mu \mathrm{g} / \mathrm{l} \mathrm{x} \mathrm{h})$ & $50.82 \pm 16.40$ & $72.61 \pm 21.21$ & $<0.01$ \\
$\mathrm{AUC}_{0-\infty}(\mu \mathrm{g} / \mathrm{l} \mathrm{h})$ & $91.63 \pm 23.20$ & $207.12 \pm 44.61$ & $<0.01$ \\
$\mathrm{t}_{1 / 2} \mathrm{Z}(\mathrm{h})$ & $1.66 \pm 0.52$ & $1.87 \pm 0.68$ & 0.280 \\
$\mathrm{~T}_{\max }(\mathrm{h})$ & $0.52 \pm 0.15$ & $0.59 \pm 0.17$ & 0.175 \\
$\mathrm{CL}_{\mathrm{z}}\left(\mathrm{m}^{3} / \mathrm{h} / \mathrm{kg}\right)$ & $2,134.0 \pm 658.2$ & $1,900.6 \pm 723.1$ & 0.291 \\
$\mathrm{C}_{\max }(\mu \mathrm{g} / \mathrm{l})$ & $43.5 \pm 14.6$ & $53.4 \pm 13.1$ & 0.030
\end{tabular}

$\mathrm{AUC}_{0-\mathrm{t}}$, area under the plasma concentration-time curve; $\mathrm{AUC}_{0-\infty}$, area under the plasma concentration-time curve extrapolated to infinity; $\mathrm{T}_{1 / 2} \mathrm{z}$, elimination half-time; $\mathrm{T}_{\max }$, time to reach the maximum plasma concentration; $\mathrm{CL}_{z} / \mathrm{F}$, clearance; $\mathrm{C}_{\max }$, maximum plasma concentration.
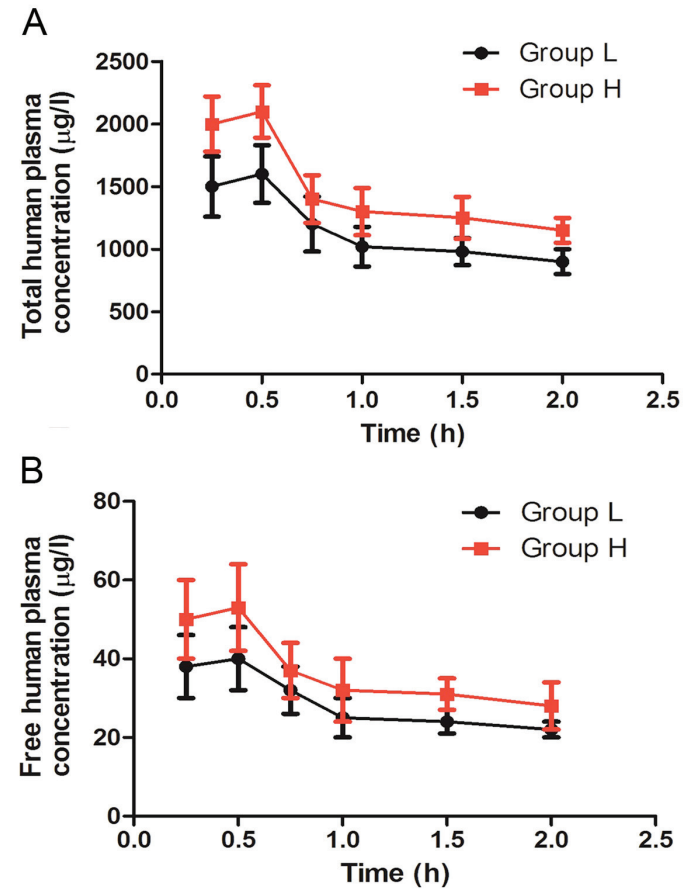

Figure 1. Concentration of (A) total plasma ropivacaine and (B) free plasma ropivacaine versus operation time.

shown in Table II, the VAS score was reduced from $6.30 \pm 0.97$ to $2.87 \pm 0.73$ in group $\mathrm{L}$, while at the same time it was reduced from $6.46 \pm 1.02$ to $2.27 \pm 0.82$ in group $\mathrm{H}(\mathrm{P}<0.05)$. Also, the VAS score was lower in group $\mathrm{H}$ than that in group $\mathrm{L}(\mathrm{P}<0.05)$ at 20 min after FICB. The lateral femoral cutaneous nerve and femoral nerve were completely blocked at $20 \mathrm{~min}$ after the operation in both groups. In group $\mathrm{H}$, the simultaneous blocking rate of lateral femoral cutaneous nerve, femoral nerve and obturator nerve was $85 \%$, similar to $75 \%$ of group $\mathrm{L}$ ( $\mathrm{P}>0.05)$, as shown in Table III. After FICB, none of the patients in either group was reported with auditory/visual deficits or articulation disorders, or other neurological problems.

Influence of pharmacokinetic parameters of ropivacaine. The results of the detection of the pharmacokinetics parameters of total and free plasma ropivacaine are shown in Tables IV and V. No detectable differences were observed in $\mathrm{t}_{1 / 2} \mathrm{z}, \mathrm{CL}_{\mathrm{z}} / \mathrm{F}$ and $\mathrm{T}_{\max }$ of exposed total and free plasma of ropivacaine between groups $\mathrm{H}$ and $\mathrm{L}(\mathrm{P}>0.05)$. The AUC of exposed total and free plasma of ropivacaine was higher in group $\mathrm{H}$ than that in group $\mathrm{L}(\mathrm{P}<0.05) . \mathrm{T}_{\max }$ of ropivacaine was $0.56 \pm 0.09$ and $0.53 \pm 0.12$ in groups $\mathrm{H}$ and $\mathrm{L}$, respectively. The total $\mathrm{C}_{\max }$ of ropivacaine was $2.17 \pm 0.56$ in group $\mathrm{H}$, higher than $1.56 \pm 0.42$ in group $\mathrm{L}(\mathrm{P}<0.05)$. The free $\mathrm{C}_{\max }$ of ropivacaine was $53.4 \pm 13.1$ in group $\mathrm{H}$, higher than $43.5 \pm 14.6$ in group $\mathrm{L}$ $(\mathrm{P}<0.05)$. There were 4 patients with total plasma concentration of ropivacaine $>2.2 \mu \mathrm{g} / \mathrm{ml}$ at some points in each group. The highest value was 3.13 and $3.34 \mu \mathrm{g} / \mathrm{ml}$ in groups $\mathrm{L}$ and $\mathrm{H}$, respectively. Neither group showed signs of central nervous system and circulatory system poisoning.

The changes of total and free plasma ropivacaine concentration were shown to be time-dependent (Fig. 1). That is, total and free plasma ropivacaine concentrations declined gradually with the prolongation of the operation time. 


\section{Discussion}

The present study was designed to evaluate the efficacy and safety of ropivacaine at different concentrations on analgesia in elderly with femoral neck fracture after FICB. The results revealed that both concentrations, 0.375 and $0.5 \%$ of ropivacaine, demonstrate a favorable effect in analgesia for FICB.

It is universally understood that the incidence of femoral neck fractures in older adults, both men and women, increases exponentially with age (30). It has been demonstrated that the systemic function of the elderly patients gradually declines, and the pain caused by the fracture decreases diagnostic accuracy while increasing therapeutic difficulty for clinicians, even leading to severe hemodynamic changes or triggering of cardiovascular and cerebrovascular events. In light of these outcomes, it is indispensable to develop early analgesia and active treatment. The FICB technique, improving the patient activities and reducing complications, is used more widely as preoperative analgesia in the elderly patients with femoral neck fractures (31). In line with the aforementioned research, our study suggests that FICB has a positively analgesic effect in elderly patients.

A previous report demonstrated that ropivacaine possesses evident postoperative analgesia capacity as reflected by the postoperative VAS score reduction (32). Consistent with this finding, our study showed that the VAS scores were obviously declined both in group L and $\mathrm{H}$ after FICB, and VAS score in group $\mathrm{H}$ was remarkably lower than that in group L. Additionally, the lateral femoral cutaneous nerve and femoral nerve were completely blocked at $20 \mathrm{~min}$ after operation in both groups. Simultaneously blocking rates of lateral cutaneous nerve, femoral nerve and obturator nerve in group $\mathrm{H}$ and L, were 85 and $75 \%$, respectively, higher than the results of Yun et al (33). Their study showed that $40 \%$ of lateral femoral cutaneous nerve, femoral nerve and obturator nerve were simultaneously blocked and $60 \%$ of lateral femoral cutaneous nerve and femoral nerve were blocked. The possible reason for this inconformity is that the blocking needle used in the study by Yun et al was $22 \mathrm{G}$, compared with the $16 \mathrm{G}$ needle of the present study, which may caused the needle to pierce the muscle group. In the present study we used ultrasound to confirm the final injection location of ropivacaine. Consistent with our data, two other studies found that ultrasound-guided FICB significantly improved the three nerve block success rate, hinting that ultrasound can improve the success rate of puncture even with a fine needle $(34,35)$. Overall, the evidence showed that a higher level of ropivacaine, remaining within the safe-dose range, influenced indexes of postoperative analgesia more intensely.

It has been suggested that a high-dose longitudinal supra-inguinal FICB with $0.5 \%$ ropivacaine enables the reduction of morphine consumption after total hip arthroplasty, although, the safety of this dose is not settled (36). Another report has shown that high plasma concentration of ropivacaine is also prone to produce a certain toxic reaction (37). In the present study, we found that the $\mathrm{C}_{\max }$ of ropivacaine was $2.17 \pm 0.56$ in group $\mathrm{H}$, higher than $1.56 \pm 0.42$ in group $\mathrm{L}$ $(\mathrm{P}<0.05)$. Over $2.2 \mu \mathrm{g} / \mathrm{ml}$ is thought to cause anesthetics poisoning (29). There were 4 patients with total plasma concentrations of ropivacaine $>2.2 \mu \mathrm{g} / \mathrm{ml}$ at some points in each group.
Neither group showed signs of central nervous system and circulatory system poisoning. The free $\mathrm{C}_{\max }$ of ropivacaine was rather low in both groups, specifically $53.4 \pm 13.1$ and $43.5 \pm 14.6$ in group $\mathrm{H}$ and $\mathrm{L}$, respectively. Besides, the $\mathrm{T}_{\max }$ was $0.56 \pm 0.09$ and $0.53 \pm 0.12$ in group $\mathrm{H}$ and $\mathrm{L}$, respectively. However, it was found that the peak concentration of 11 patients appeared in $15 \mathrm{~min}$, due to the experimental design, and no point before 15 min was chosen. There may be some bias in the pharmacokinetic analysis of DAS 3.0, and therefore the peak value of drugs may occur earlier. The rich vascular muscle between iliac fascia space may lead to rapid absorption, suggesting that high dose and high-dose injection between iliac fascia space may bring the risk of local anesthetics poisoning. Different from the results of Paut et al (22), reported in pediatric FICB, the results of the present study revealed that the maximum concentration occurred at 20-90 min. The heart and nervous system toxicity from the free section, and the plasma concentrations of ropivacaine to induce toxic reaction is still not unified. In spite of this, it is realized that the concentration of $0.5 \%$ ropivacaine remains a risk for further extensive analgesia treatment based on our data. However, our results are based on a small sample size without young subjects as control, so the difference between the elderly and young people is unclear. Sampling time also needs further study. For elder patients, the most important factor involved in drug pharmacokinetics is the decreased liver function.

In conclusion, $0.375 \%$ and $0.5 \%$ of ropivacaine have a favourable effect in analgesia for FICB. Nonetheless, there is a theoretical risk of local anesthetics poisoning in both groups, suggesting that a lower dose might be a good choice for a single large volume of FICB.

\section{Acknowledgements}

Not applicable.

\section{Funding}

This study was supported by the Projects of Medical and Health Technology Development Program in Zhejiang Province (no. 2014kyb).

\section{Availability of data and materials}

The datasets used during the present study are available from the corresponding author upon reasonable request.

\section{Authors' contributions}

XWG and FFZ designed the study, wrote, drafted and revised the manuscript. CL and LYY performed the experiments. FFZ, SPW and MZ collected the samples, analyzed the data and made the diagrams. All authors read and approved the final manuscript.

\section{Ethics approval and consent to participate}

The study was approved by the Ethics committee of the First Affiliated Hospital of Zhejiang Chinese Medicine University (Hangzhou, China) (no. 2013-k-058). All patients were informed of the study and signed a consent form. 


\section{Patient consent for publication}

Not applicable.

\section{Competing interests}

The authors declare that they have no competing interests.

\section{References}

1. Wiffen PJ, Derry S, Moore RA, McNicol ED, Bell RF, Carr DB, McIntyre M and Wee B: Oral paracetamol (acetaminophen) for cancer pain. Cochrane Database Syst Rev 7: CD012637, 2017.

2. Aghazadeh-Habashi A, Asghar W and Jamali F: Drug-disease interaction: Effect of inflammation and nonsteroidal anti-inflammatory drugs on cytochrome $\mathrm{P} 450$ metabolites of arachidonic acid. J Pharm Sci 107: 756-763, 2018.

3. Sostres C, Carrera-Lasfuentes P and Lanas A: Non-steroidal anti-inflammatory drug related upper gastrointestinal bleeding: Types of drug use and patient profiles in real clinical practice. Curr Med Res Opin 33: 1815-1820, 2017.

4. Ostrowska H: Inhibition of human platelet cathepsin A by non-steroidal anti-inflammatory drugs - in vitro study. Pol J Pharmacol 48: 113-116, 1996.

5. Scharf RE: Drugs that affect platelet function. Semin Thromb Hemost 38: 865-883, 2012

6. Nicholson BD: Economic and clinical burden of opioid-induced nausea and vomiting. Postgrad Med 129: 111-117, 2017.

7. Fox LM, Hoffman RS, Vlahov D and Manini AF: Risk factors for severe respiratory depression from prescription opioid overdose. Addiction 113: 59-66, 2018

8. Gomes T, Juurlink DN, Antoniou T, Mamdani MM, Paterson JM and van den Brink W: Gabapentin, opioids, and the risk of opioid-related death: A population-based nested case-control study. PLoS Med 14: e1002396, 2017.

9. Webster LR: Risk factors for opioid-use disorder and overdose. Anesth Analg 125: 1741-1748, 2017.

10. Kenes MT, Stollings JL, Wang L, Girard TD, Ely EW and Pandharipande PP: Persistence of delirium after cessation of sedatives and analgesics and impact on clinical outcomes in critically Ill patients. Pharmacotherapy 37: 1357-1365, 2017.

11. Capdevila X, Biboulet P, Bouregba M, Barthelet Y, Rubenovitch J and d'Athis F: Comparison of the three-in-one and fascia iliaca compartment blocks in adults: Clinical and radiographic analysis. Anesth Analg 86: 1039-1044, 1998.

12. Tran DQ, Clemente A and Finlayson RJ: A review of approaches and techniques for lower extremity nerve blocks. Can J Anaesth 54: 922-934, 2007.

13. Eastburn E, Hernandez MA and Boretsky K: Technical success of the ultrasound-guided supra-inguinal fascia iliaca compartment block in older children and adolescents for hip arthroscopy. Paediatr Anaesth 27: 1120-1124, 2017.

14. Odor PM, Chis Ster I, Wilkinson I and Sage F: Effect of admission fascia iliaca compartment blocks on post-operative abbreviated mental test scores in elderly fractured neck of femur patients: A retrospective cohort study. BMC Anesthesiol 17: 2, 2017.

15. Pinson S: Fascia Iliaca (FICB) block in the emergency department for adults with neck of femur fractures: A review of the literature. Int Emerg Nurs 23: 323-328, 2015.

16. Chesters A and Atkinson P: Fascia iliaca block for pain relief from proximal femoral fracture in the emergency department: a review of the literature. Emerg Med J 31: e84-87, 2014.

17. Mittal R and Vermani E: Femoral nerve blocks in fractures of femur: Variation in the current UK practice and a review of the literature. Emerg Med J 31: 143-147, 2014.

18. Satsumae T, Tanaka M, Saito S and Inomata S: Convulsions after ropivacaine $300 \mathrm{mg}$ for brachial plexus block. Br J Anaesth 101: 860-862, 2008

19. Hansen TG: Ropivacaine: A pharmacological review. Expert Rev Neurother 4: 781-791, 2004

20. Das NT and Deshpande C: Effects of intraperitoneal local anaesthetics bupivacaine and ropivacaine versus placebo on postoperative pain after laparoscopic cholecystectomy: A randomised double blind study. J Clin Diagn Res 11: UC08-UC12, 2017.
21. Rao KG, Misra S and Shukla A: Comparison between epidural ropivacaine versus ropivacaine with clonidine in patients undergoing abdominal hysterectomy: A randomized study. Anesth Essays Res 11: 334-339, 2017.

22. Paut O, Schreiber E, Lacroix F, Meyrieux V, Simon N, Lavrut T, Camboulives $\mathbf{J}$ and Bruguerolle $\mathrm{B}$ : High plasma ropivacaine concentrations after fascia iliaca compartment block in children. Br J Anaesth 92: 416-418, 2004.

23. Dhir S, Ganapathy S, Lindsay P and Athwal GS: Case report: Ropivacaine neurotoxicity at clinical doses in interscalene brachial plexus block. Can J Anaesth 54: 912-916, 2007.

24. Sung HJ, Ok SH, Sohn JY, Son YH, Kim JK, Lee SH, Han JY, Lim DH, Shin IW, Lee HK, et al: Vasoconstriction potency induced by aminoamide local anesthetics correlates with lipid solubility. J Biomed Biotechnol 2012: 170958, 2012.

25. Xiao J, Cai MH, Wang XR, He P and Wang XR: Time course of action and pharmacokinetics of ropivacaine in adult and elderly patients following combined lumbar plexus-sciatic nerve block. Int J Clin Pharmacol Ther 48: 608-613, 2010.

26. Torup H, Mitchell AU, Breindahl T, Hansen EG, Rosenberg J and Møller AM: Potentially toxic concentrations in blood of total ropivacaine after bilateral transversus abdominis plane blocks; a pharmacokinetic study. Eur J Anaesthesiol 29: 235-238, 2012.

27. Koköfer A, Nawratil J, Felder TK, Stundner O, Mader N and Gerner P: Ropivacaine $0.375 \%$ vs. $0.75 \%$ with prilocaine for intermediate cervical plexus block for carotid endarterectomy: A randomised trial. Eur J Anaesthesiol 32: 781-789, 2015.

28. Peng PW, Coleman MM, McCartney CJ, Krone S, Chan VW, Kaszas Z and Vucemilo I: Comparison of anesthetic effect between $0.375 \%$ ropivacaine versus $0.5 \%$ lidocaine in forearm intravenous regional anesthesia. Reg Anesth Pain Med 27: 595-599, 2002.

29. Sun N, Wang S, Ma P, Liu S, Shao A and Xiong L: Postoperative analgesia by a transversus abdominis plane block using different concentrations of ropivacaine for abdominal surgery: A meta-analysis. Clin J Pain 33: 853-863, 2017.

30. Senohradski K, Markovic-Denic L, Lesic A, Bumbasirevic V and Bumbasirevic M: Trends in the incidence of hip fractures. Osteoporosis Int 24: 1759-1763, 2013.

31. Miller GW, Godrey JJ, Sagmeister ML and Lewis TL: Provision of fascia iliaca compartment block in the acute management of proximal femoral fractures: A national observational study of UK hospitals. Injury 47: 2490-2494, 2016.

32. Su Y, Zhang Z, Zhang Y, Li H and Shi W: Efficacy of ropivacaine by the concentration of $0.25 \%, 0.5 \%$, and $0.75 \%$ on surgical performance, postoperative analgesia, and patient's satisfaction in inguinal hernioplasty: A randomized controlled trial. Patient Prefer Adherence 9: 1375-1379, 2015.

33. Yun MJ, Kim YH, Han MK, Kim JH, Hwang JW and Do SH: Analgesia before a spinal block for femoral neck fracture: Fascia iliaca compartment block. Acta Anaesthesiol Scand 53: 1282-1287, 2009.

34. Kumar D, Hooda S, Kiran S and Devi J: Analgesic efficacy of ultrasound guided FICB in patients with hip fracture. J Clin Diagn Res 10: UC13-UC16, 2016.

35. Dolan J, Williams A, Murney E, Smith M and Kenny GN: Ultrasound guided fascia iliaca block: A comparison with the loss of resistance technique. Reg Anesth Pain Med 33: 526-531, 2008.

36. Desmet M, Vermeylen K, Van Herreweghe I, Carlier L, Soetens F, Lambrecht S, Croes K, Pottel H and Van de Velde M: A longitudinal supra-inguinal fascia iliaca compartment block reduces morphine consumption after total hip arthroplasty. Reg Anesth Pain Med 42: 327-333, 2017.

37. Graf BM, Abraham I, Eberbach N, Kunst G, Stowe DF and Martin E: Differences in cardiotoxicity of bupivacaine and ropivacaine are the result of physicochemical and stereoselective properties. Anesthesiology 96: 1427-1434, 2002.

This work is licensed under a Creative Commons Attribution-NonCommercial-NoDerivatives 4.0 International (CC BY-NC-ND 4.0) License. 